\title{
Two Cases of Mitochondrial Diabetes in Which Pancreatic Beta-Cell Function and Neuropathy Were Improved by Glucagon-Like Peptide-1 Receptor Agonist Therapy
}

\author{
Midori Minezaki ${ }^{a}$, Ichiro Abe ${ }^{\text {a, d }}$, Monami Koga ${ }^{a}$, Kaoru Sugimoto ${ }^{\text {a }}$ Kaori Takeshita ${ }^{\text {a }}$ \\ Saori Takahara ${ }^{a}$, Midori Nakagawa ${ }^{\text {a }}$, Hideyuki Fujii ${ }^{\mathrm{a}}$, Hanako Ohishi ${ }^{\mathrm{a}}$, \\ Tadachika Kudo ${ }^{a}$, Kenji Ohe ${ }^{b}$, Toshihiko Yanase ${ }^{c}$, \\ Kunihisa Kobayashi ${ }^{\mathrm{a}}$
}

\begin{abstract}
Mitochondrial diabetes is a refractory type of diabetes mellitus where pancreas beta-cell function attenuates. Glucagon-like peptide-1 receptor agonists (GLP-1RAs) has been reported for pleiotropic effects in addition to effect to glucose tolerance. In animal models, GLP1RAs were reported to recover mitochondrial function of pancreas beta-cells and increase the growth rate of nerve cells. Our two cases appear to be the first reported cases in the literature of mitochondrial diabetes where GLP-1RAs improved insulin secretion and neuropathy.
\end{abstract}

Keywords: Mitochondrial diabetes; Glucagon-like peptide-1 receptor agonists; Pancreatic beta-cell function; Neuropathy

\section{Introduction}

Mitochondrial loss of function caused by abnormalities in the mitochondrial DNA (mtDNA) can lead to impairments in nerve and muscle function, but also in pancreatic beta-cell function. The former is referred to as MELAS (mitochondrial myopathy, encephalopathy, lactic acidosis, stroke-like episodes), and the latter as mitochondrial diabetes $[1,2]$. It has been reported that $2 \%$ of cases of diabetes mellitus in Japan are due to mtDNA mutations, making it the most common genetic

Manuscript submitted November 22, 2018, accepted November 29, 2018

aDepartment of Endocrinology and Diabetes Mellitus, Fukuoka University Chikushi Hospital, Japan

${ }^{b}$ Department of Pharmacotherapeutics, Faculty of Pharmaceutical Sciences, Fukuoka University, Japan

'Department of Endocrinology and Diabetes Mellitus, Faculty of Medicine, Fukuoka University, Japan

${ }^{\mathrm{d}}$ Corresponding Author: Ichiro Abe, Department of Endocrinology and Diabetes Mellitus, Fukuoka University Chikushi Hospital, 1-1-1 Zokumyoin, Chikushino, Fukuoka 818-8502, Japan. Email: abe1ro@fukuoka-u.ac.jp

doi: https://doi.org/10.14740/jem539 etiology of diabetes mellitus [3]. The neuropathy associated with mitochondrial diabetes tends to be more severe than when it occurs as a single diabetic complication, because it develops alongside MELAS.

Insulin therapy is usually prescribed for patients with mitochondrial diabetes [4]. However, glucagon-like peptide-1 receptor agonists (GLP-1Ras) have been reported to improve pancreatic beta-cell function [5] and neuropathy [6]. Therefore, we investigated the feasibility of using GLP1RAs for the treatment of mitochondrial diabetes with neuropathy.

\section{Case Reports}

\section{Case 1}

Case 1 was a 36-year-old woman who had been originally hospitalized due to a high blood glucose level $(458 \mathrm{mg} / \mathrm{dL})$ at a regular health check when she was 27 years old. Because her mother had been diagnosed with MELAS and she was experiencing slight sensorineural hearing loss, she underwent a genetic evaluation. She was diagnosed with mitochondrial diabetes on the basis of a mitochondrial 3243A $>$ G mutation. Her blood glucose control was poor, with frequent hypoglycemic events, and she was hospitalized to improve this. Her clinical and laboratory data on admission are shown in Table 1. Her hemoglobin A1c (HbAlc) was $8.1 \%$ despite taking daily 42 units of insulin. Her glucometabolic data showed attenuated insulin secretion and a high lactate/pyruvate ratio, indicative of predominant anaerobic metabolism. She also demonstrated paresthesia in both legs such as reduced vibration sensation examined by a $\mathrm{C} 128$ tuning fork (right $8 \mathrm{~s}$, left $9 \mathrm{~s}$ (normal $>10$ s)) and numbness. Her clinical and laboratory data are shown in Table 1. After admission, liraglutide was administered and her insulin dose was appropriately adjusted at this time and after discharge. One year later, her $\mathrm{HbA} 1 \mathrm{c}$ decreased to $7.4 \%$, daily insulin dose was 15 units, and C-peptide index (CPI: fasting C-peptide/fasting plasma glucose $\times 100$ ) increased to 1.27 , implying improved insulin secretion. The vibration sensation of both legs improved to $13 \mathrm{~s}$ and numbness in both lower 
Table 1. Clinical Characteristics of the Patients

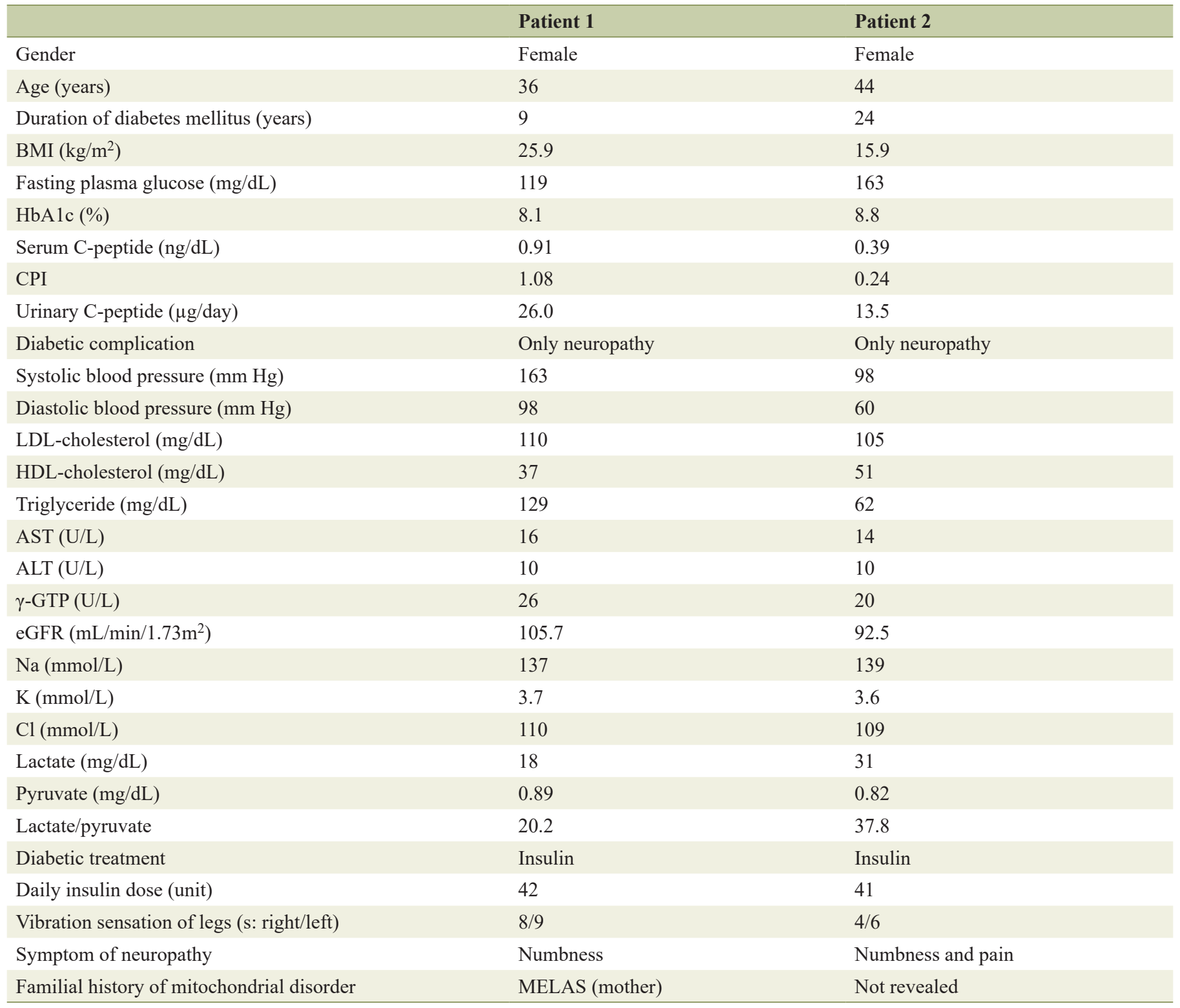

CPI: fasting C-peptide/fasting blood glucose $\times 100$; eGFR: estimated glomerular filtration rate.

limbs had disappeared (Table 2).

\section{Case 2}

Case 2 was a 44-year-old woman who had been diagnosed with abnormal glucose tolerance when 20 years old. Although detailed information was not available, she had been diagnosed with type 1 diabetes mellitus and neurological disorders were identified at around 30 years of age. Insulin therapy had been commenced, but her blood glucose was unstable. When she first attended our clinic hearing loss was diagnosed and genetic screening was conducted, which identified a mitochondrial $3243 \mathrm{~A}>\mathrm{G}$ mutation, leading to a diagnosis of mitochondrial diabetes. Her HbA1c was high (8.8\%, despite taking daily 41 units of insulin) and she was hospitalized to improve her blood glucose control. Her clinical and laboratory data on admission are shown in Table 1. Her glucometabolic data indicated severely attenuated insulin secretion, and the high lactate/pyruvate ratio of 37.8 was indicative of predominant anaerobic metabolism. She also demonstrated paresthesia in both legs such as reduced vibration sensation also demonstrated low vibration sensation in both legs examined by a C128 tuning fork (right $4 \mathrm{~s}$, left $6 \mathrm{~s}$ ), numbness, and pain in both lower legs and feet. After admission, liraglutide was administered and her insulin dose was adjusted, which was continued after discharge. One year later her $\mathrm{HbAlc}$ decreased to $8.2 \%$ and $\mathrm{C}$-peptide index (CPI) increased to 0.48 alongside reduction of daily insulin 
Table 2. Glycemic Parameters and Symptoms Before and 1 Year After Commencing Liraglutide Therapy

\begin{tabular}{|lll}
\hline & Before commencing liraglutide & One year after commencing liraglutide \\
\hline Patient 1 & & \\
\hline Fasting plasma glucose $(\mathrm{mg} / \mathrm{dL})$ & 119 & 114 \\
HbAlc (\%) & 8.1 & 7.4 \\
CPI & 1.08 & 1.27 \\
\hline Daily insulin dose (unit) & 42 & 15 \\
Daily liraglutide dose (mg) & 0 & 0.9 \\
Vibration sensation of legs (s: right/left) & $8 / 9$ & $13 / 13$ \\
Symptom of neuropathy & Numbness & None \\
Patient 2 & & \\
Fasting plasma glucose (mg/dL) & 163 & 111 \\
HbA1c (\%) & 8.8 & 8.2 \\
CPI & 0.24 & 0.49 \\
Daily insulin dose (unit) & 41 & 14 \\
Daily liraglutide dose (mg) & 0 & 0.9 \\
Vibration sensation of legs (s: right/left) & $4 / 6$ & $8 / 8$ \\
Symptom of neuropathy & Numbness and pain & Slight pain \\
\hline
\end{tabular}

CPI: fasting C-peptide/fasting blood glucose $\times 100$.

dose (from 41 to 14 units), implying improved insulin secretion. The vibration sensation in both legs improved to $8 \mathrm{~s}$, the numbness in both lower limbs disappeared, and her pain improved markedly (Table 2).

Two participants provided written informed consent about publication of this case report, which was approved by the Ethics Review Committee of Fukuoka University (Japan).

\section{Discussion}

Abnormalities in mtDNA can lead to mitochondrial loss of function and thus impairments in muscle and nerve function. MELAS, first reported by Pavlakis et al [1], is the most commonly reported disease-causing mtDNA abnormality, involving a $3243 \mathrm{~A}>\mathrm{G}$ mutation in the tRNALeu (UUR) (mitochondrially encoded tRNA leucine 1) gene [7]. However, the same mutation has been found in individuals with maternally inherited type 2 diabetes mellitus and sensorineural deafness [2], which are currently diagnosed as having mitochondrial diabetes. mtDNA abnormalities tend to cause disease because of the abundance of mtDNA: there is an average of several hundred mitochondria per cell, with 2 - 10 mtDNA copies each, making several thousand mtDNA copies per cell. A pathogenic mtDNA mutation that coexists with normal mtDNA is referred to as heteroplasmy, and the resultant variability in the symptoms is thought to depend on the ratio of normal: abnormal sequences. Mutant and wild-type mtDNA is found in different ratios in different organs and even within different cells of the same organ. Thus, when the nerves and muscles are mainly affected, MELAS develops, whereas when pancreatic beta-cells are affected, mitochondrial diabetes develops [8].
Recent researches into the extra-pancreatic functions of incretins have shown possible beneficial effects on diabetic microangiopathy [9]. Moreover, GLP-1RAs have been reported to ameliorate pancreatic beta-cell loss by reducing apoptosis in animal models, in addition to their effect to increase insulin secretion [5]. The two patients with mitochondrial diabetes reported here showed improved insulin secretion after the administration of GLP-1RAs, implying a beneficial effect of GLP-1RAs on their pancreatic beta-cell function. In addition, GLP-1RAs have been reported to ameliorate the loss of nerve cells [10] and to improve neuropathy [6] in animal models. The results obtained with our patients indicate the possibility that GLP-1RAs may alleviate neuropathies in humans, even in patients with mitochondrial diabetes as a part of MELAS.

In conclusion, we report here two patients with mitochondrial diabetes in whom GLP-1RA therapy improved pancreatic beta-cell function and ameliorated neuropathy. Additional studies will be necessary to further evaluate the use of GLP1 RAs as a novel therapeutic approach for mitochondrial diabetes.

\section{Acknowledgments}

We thank Mr. Hideaki Shimada and Ms. Yumi Iriguchi for assistance.

\section{Conflict of Interest}

All authors declare that there is no conflict of interests regarding the publication of this paper. Toshihiko Yanase was sup- 
ported financially in his research by MSD, Sanofi, Takeda, Daiichi Sankyo, Sumitomo Dainippon, Sanwa Chemistry, Eli Lilly Japan, Novo Nordisk, Novartis, Kowa, Boehringer Ingelheim, and Fujifilm. Kunihisa Kobayashi received honoraria from Mitsubishi-Tanabe, Ono, and MSD.

\section{References}

1. Pavlakis SG, Phillips PC, DiMauro S, De Vivo DC, Rowland LP. Mitochondrial myopathy, encephalopathy, lactic acidosis, and strokelike episodes: a distinctive clinical syndrome. Ann Neurol. 1984;16(4):481-488.

2. van den Ouweland JM, Lemkes HH, Ruitenbeek W, Sandkuijl LA, de Vijlder MF, Struyvenberg PA, van de Kamp JJ, et al. Mutation in mitochondrial tRNA(Leu) (UUR) gene in a large pedigree with maternally transmitted type II diabetes mellitus and deafness. Nat Genet. 1992;1(5):368-371.

3. Kadowaki T, Kadowaki H, Mori Y, Tobe K, Sakuta R, Suzuki Y, Tanabe Y, et al. A subtype of diabetes mellitus associated with a mutation of mitochondrial DNA. N Engl J Med. 1994;330(14):962-968.

4. Shigemoto M, Yoshimasa Y, Yamamoto Y, Hayashi T, Suga J, Inoue G, Okamoto M, et al. Clinical manifestations due to a point mutation of the mitochondrial
tRNAleu(UUR) gene in five families with diabetes mellitus. Intern Med. 1998;37(3):265-272.

5. Ogata M, Iwasaki N, Ide R, Takizawa M, Uchigata Y. GLP-1-related proteins attenuate the effects of mitochondrial membrane damage in pancreatic beta cells. Biochem Biophys Res Commun. 2014;447(1):133-138.

6. Himeno T, Kamiya H, Naruse K, Harada N, Ozaki N, Seino Y, Shibata T, et al. Beneficial effects of exendin-4 on experimental polyneuropathy in diabetic mice. Diabetes. 2011;60(9):2397-2406.

7. Goto Y, Nonaka I, Horai S. A mutation in the tRNA(Leu) (UUR) gene associated with the MELAS subgroup of mitochondrial encephalomyopathies. Nature. 1990;348(6302):651-653.

8. Onishi $\mathrm{H}$, Inoue $\mathrm{K}$, Osaka $\mathrm{H}$, Kimura $\mathrm{S}$, Nagatomo $\mathrm{H}$, Hanihara T, Kawamoto S, et al. Mitochondrial myopathy, encephalopathy, lactic acidosis and stroke-like episodes (MELAS) and diabetes mellitus: molecular genetic analysis and family study. J Neurol Sci. 1993;114(2):205-208.

9. Avogaro A, Fadini GP. The effects of dipeptidyl peptidase-4 inhibition on microvascular diabetes complications. Diabetes Care. 2014;37(10):2884-2894.

10. Parthsarathy V, Holscher C. Chronic treatment with the GLP1 analogue liraglutide increases cell proliferation and differentiation into neurons in an AD mouse model. PLoS One. 2013;8(3):e58784. 